\title{
Use of concrete formulations based on dredging sand in the fabrication of tetrapods for protection of harbour dykes
}

\author{
Mehdi HASSOUNE ${ }^{1}$, Hassan FATMAOUI ${ }^{1}$ and Jamal CHAOUFI ${ }^{1}$ \\ ${ }^{I}$ University of Sciences Ibn Zohr Agadir, Morocco
}

Keywords: Concrete; dredged sand; tetrapods; dikes; environment.

Abstract: Port structures (quays and dykes) need an enormous consumption of materials, in particular concrete and its components, as well as sand, hence the need to develop alternative solutions using port dredging sand, estimated at thousands of tonnes through its use in the concrete that forms the blocks of the quay walls. After having demonstrated in a previous work the possibility of using the dredged sand from the port of Agadir in concrete formulations, the present work consists in showing the possibility of using the dredged sand from the port of Agadir in concrete formulations that can be used in the construction of concrete tetrapods from a concrete formulation based on dredged sand, more precisely $50 \%$ of the sand entering in the formulation is dredged sand extracted from the port of Agadir. The eventual use of dredged sand in the concrete of the tetrapods will lead to enormous gains in the quantities of sand and thus to a lower consumption of construction materials, and will also play a more important role in the preservation of the marine environment by avoiding the trapping of the dredged materials.

\section{INTRODUCTION}

Wave protection structures are structures that protect a port or part of a port against the actions of waves (direct attack, crossings, submersion, erosion, etc.). They are man-made structures, except for natural structures which may have the same functions, among these structures are tetrapod dikes, which are structures that provide protection against waves by placing tetrapods in a shell and a core made of riprap. Tetrapods are four-legged structures, used particularly in hydraulic engineering as part of breakwaters to resist waves and swells.

Typically, tetrapods are made of concrete, not reinforced, and have four members with a curved profile designed to minimize the impact of marine flows. The design of the structure allows the blocks to be interlocked, which, when used in masses, allows coastal protection to be reinforced or even to gain surface areas out of the water on the sea.

The use of tetrapods in particular is very widespread, and their fabrication requires large quantities of concrete and subsequently large quantities of sand, which is why it was necessary to look for alternative solutions to substitute sand and why it is proposed subsequently to replace the sand in the concrete formulations with dredging sand extracted from the port of Agadir. By analysing the different constituents of the concrete formulations studied afterwards, as well as the results obtained, we will conclude on the possibility of finding concrete formulations based on dredging sand able to be used in concrete for tetrapods.

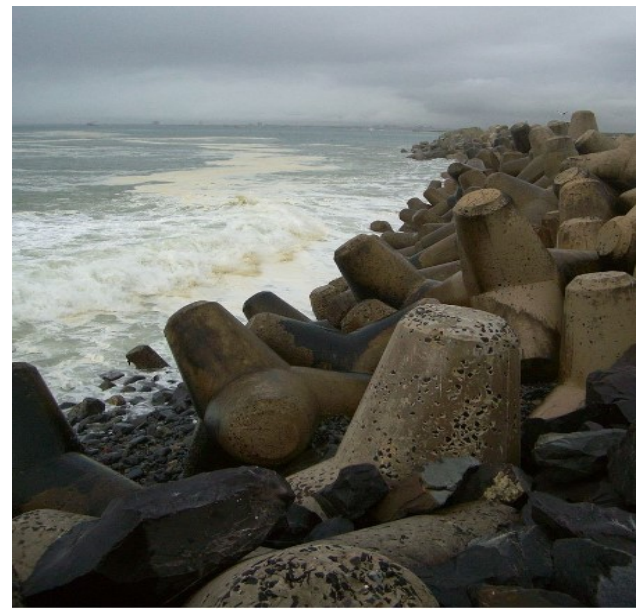

Figure 1: Tetrapod breakwater for protection against swells 


\section{THE STUDY OF THE CONCRETE COMPOSITION OF TETRAPODS BASED ON THE DREDGED SAND OF THE PORT OF AGADIR}

\subsection{Origin of materials}

The materials used in the fabrication of tetrapods concrete from the dredged sand of the port of Agadir are composed of :

- Crushed aggregates from the Ynoub quarry located in Ighoud in the Agadir region;

- Dredging sand from the port of Agadir;

- Crushing sand;

- Cement CPJ55PM from the CIMAR Aït Baha plant in the Agadir region;

- Viscocrete Tempo 1000M admixtures from the company SIKA MAROCCO;

- Mixing water which is well water.

\subsection{Characteristics and materials performance testing}

\subsubsection{Aggregates}

The values of the intrinsic and production characteristics of the aggregates are given in the table below:

Table 1: Characteristics of the aggregates

\begin{tabular}{|c|c|c|c|}
\hline \multicolumn{4}{|c|}{ Identification of gravel } \\
\hline Samples & Origin & $\begin{array}{c}\text { flattening } \\
\text { factor \% }\end{array}$ & Cleanliness \\
\hline GI & Ech 1 & $15<30$ & $0,9<1,5$ \\
\hline GII & Ech 1 & $17<30$ & $0,8<1,5$ \\
\hline
\end{tabular}

\subsubsection{Sands}

The characteristics of the sands are given in the following tables

Table 2: CHARACTERISTICS OF THE SANDS

\begin{tabular}{|c|c|c|c|c|}
\hline Samples & $\begin{array}{c}\text { Dma } \\
\mathrm{x} \\
\mathrm{mm}\end{array}$ & $\begin{array}{c}\%<0,08 \\
0 \mathrm{~mm}\end{array}$ & $\begin{array}{c}\text { Sand } \\
\text { equivale } \\
\text { nt (SE) } \\
\%\end{array}$ & $\begin{array}{c}\text { Finenes } \\
\mathrm{s} \\
\text { modulu } \\
\mathrm{s}\end{array}$ \\
\hline $\begin{array}{c}\text { Dredgin } \\
\mathrm{g} \text { sand }\end{array}$ & 1,6 & 0,9 & - & - \\
\hline
\end{tabular}

\begin{tabular}{|c|c|c|c|c|}
\hline $\begin{array}{c}\text { Crushin } \\
\text { g sand }\end{array}$ & 8,0 & 11,2 & - & - \\
\hline $\begin{array}{c}\text { Mixing } \\
\text { ( } 50 \%\end{array}$ & 6,3 & 6,6 & 80 & 2,46 \\
dredgin & & & & \\
g sand + & & & & \\
$50 \%$ & & & & \\
crushing & & & & \\
sand) & & & & \\
\hline
\end{tabular}

\subsubsection{Other tests carried out on the aggregates and sands of the studied formulation}

Laboratory tests were also carried out for the GI and GII aggregates, the crushing sand and the dredging sand from the port of Agadir in order to enable us to make a preliminary decision on the effectiveness of the tetrapod concrete formulation studied.

The autoclave test carried out on the mixture made up of $50 \%$ crushed sand and $50 \%$ dredging sand qualified the mixture as nonreactive (NR).

Table 3: General Characteristics of AGGREgATES AND SAND

\begin{tabular}{|c|c|c|c|c|c|}
\hline Designation & $\begin{array}{c}\text { GII } \\
16 / 31.5\end{array}$ & $\begin{array}{c}\text { GI } \\
4 / 16\end{array}$ & $\mathrm{SC} 0 / 4$ & SD & $\begin{array}{c}\text { Standar } \\
\mathrm{d}\end{array}$ \\
\hline $\begin{array}{c}\text { Flattening } \\
\text { A }(\%)\end{array}$ & 21 & 20 & \multicolumn{2}{|c|}{--} & $<25 *$ \\
\hline $\begin{array}{c}\text { Absorption } \\
\mathrm{Ab}(\%)\end{array}$ & 0.5 & 0.8 & 0.8 & 0.6 & $<2.5 *$ \\
\hline $\begin{array}{c}\text { Cleanliness } \\
(\%)\end{array}$ & 0.2 & 1.1 & \multicolumn{2}{|c|}{--} & $<1.5 *$ \\
\hline $\begin{array}{c}\text { ESV } \\
\text { mixture * } \\
(\%)\end{array}$ & -- & -- & \multicolumn{2}{|c|}{79} & $\begin{array}{c}\mathrm{ESV}> \\
75 \text { ou } \\
\mathrm{VB}<1\end{array}$ \\
\hline $\begin{array}{l}\text { Mf of } \\
\text { mixture* } \\
(\%)\end{array}$ & -- & -- & \multicolumn{2}{|c|}{2.1} & $\begin{aligned} 2 & <\mathrm{Mf} \\
& <3\end{aligned}$ \\
\hline $\begin{array}{l}\text { Content of } \\
\text { fines } F^{*}(\%)\end{array}$ & -- & -- & \multicolumn{2}{|c|}{7.8} & $\leq 12$ \\
\hline $\begin{array}{c}\text { Organic } \\
\text { materials }\end{array}$ & -- & -- & \multicolumn{2}{|c|}{ - } & negatif \\
\hline $\begin{array}{c}\text { Alkali } \\
\text { reaction }\end{array}$ & \multicolumn{2}{|c|}{ NR } & \multicolumn{2}{|c|}{ NR } & C Level \\
\hline
\end{tabular}

Table 4: Autoclave test Results

\begin{tabular}{|c|c|c|c|}
\hline Tests & Value \% & $\begin{array}{c}\text { Requirement } \\
\%\end{array}$ & $\begin{array}{c}\text { Test } \\
\text { method }\end{array}$ \\
\hline Alkali & 0,06 & 0,15 & NM \\
\hline
\end{tabular}




\begin{tabular}{|c|l|l|}
\hline $\begin{array}{c}\text { reaction ( } \\
\text { Expansion in } \\
\% \mathrm{C} / \mathrm{G}=0.5)\end{array}$ & & 10.1 .279 \\
\hline
\end{tabular}

The expansion value obtained on the mixture $(50 \% \mathrm{SC}+50 \% \mathrm{SD})$ is lower than the maximum limit value set by the standard NM 10.1.271, version 2008. Therefore, this mixture is nonreactive with respect to the alkali reaction (NR).

\subsubsection{Cement}

The cement used is CPJ55 Prise Mer with limestone filler manufactured by the CIMAR plant located in Aït Baha-Agadir. The summary of the results of the cement self-checking tests is attached below. The cement used for the study formulas has a resistance at 28 days is $51.7 \mathrm{MPa}$.

Table 5: Cement test RESults CPJ 55

\begin{tabular}{|c|c|c|c|c|c|c|}
\hline & p.a.f & $\mathrm{MgO}$ & $\mathrm{SO} 3$ & $\mathrm{Na} 2 \mathrm{O}$ & $\mathrm{K} 2 \mathrm{O}$ & $\mathrm{Cl}$ \\
\hline Average & 5,98 & 1,58 & 2,85 & 0,12 & 1,47 & 0,01 \\
\hline $\begin{array}{c}\text { Standard } \\
\text { deviation }\end{array}$ & 0,60 & 0,25 & 0,14 & 0,04 & 0,13 & 0,0 \\
\hline Max & 6,98 & 1,96 & 3,09 & 0,17 & 1,81 & 0,02 \\
\hline Min & 4,72 & 0,92 & 2,61 & 0,0 & 1,29 & 0,01 \\
\hline
\end{tabular}

\begin{tabular}{|c|c|c|c|c|c|c|c|}
\hline & $\begin{array}{c}\text { Insoluble } \\
\text { dans HCl }\end{array}$ & SSB & $\%$ water & $\begin{array}{c}\text { Start of } \\
\text { setting }\end{array}$ & RC2J & RC7J & RC28J \\
\hline Average & 0,96 & 4100,70 & 28,17 & 183,38 & 29,90 & 40,05 & 50,03 \\
\hline $\begin{array}{c}\text { Standard } \\
\text { deviation }\end{array}$ & 0,09 & 191,15 & 0,25 & 8,92 & 2,01 & 1,78 & 1,97 \\
\hline Max & 1,14 & 4559,0 & 28,70 & 198,0 & 32,60 & 44,20 & 52,90 \\
\hline Min & 0,85 & 3807,0 & 27,70 & 165,0 & 25,30 & 36,60 & 45,60 \\
\hline
\end{tabular}

\subsubsection{Admixture}

The admixture used for this concrete is Sika Maroc's Viscocrète Tempo $1000 \mathrm{M}$, which is a new generation superplasticizer with high water reducer properties.

In self-compacting concretes, SIKA VISCOCRETE TEMPO $1000 \mathrm{M}$ improves stability, limits concrete segregation and makes the formulas less susceptible to variations in water and constituents.
SIKA VISCOCRETE TEMPO $1000 \mathrm{M}$ allows a significant reduction of the $\mathrm{W} / \mathrm{C}$ ratio which improves the durability of the hardened concrete (reduced permeability, increased mechanical resistance, reduced shrinkage).

SIKA VISCOCRETE® TEMPO $1000 \mathrm{M}$ does not contain chlorides or other substances that may cause or promote chloride reactions.

The table below lists the characteristics taken from the technical data sheet:

Table 6: CHARACTERISTICS OF ADMIXTURE

\begin{tabular}{|c|c|}
\hline Designation & Technical Data Sheet \\
\hline Extrait Sec & $28.5 \pm 1.5 \%$ \\
\hline PH & $4 \pm 1$ \\
\hline Alcalins & $\leq 1 \%$ \\
\hline Density & $1.07 \pm 0.02$ \\
\hline
\end{tabular}

\subsubsection{Compatibility tests Admixture and Concrete Constituents}

Concrete formulations were studied by analyzing the change and variation in the percentage of admixture in the concrete formulation.

The table below summarizes the results found:

\begin{tabular}{|c|c|c|c|c|c|}
\hline \multirow{3}{*}{$\begin{array}{c}\text { Design } \\
\text { ation }\end{array}$} & \multicolumn{2}{|c|}{$\begin{array}{l}\text { Table 7: } \\
\text { ADMIXTURE } \\
\text { CONSTITUENTS }\end{array}$} & \multicolumn{2}{|c|}{$\begin{array}{l}\text { COMPATIBILITY } \\
\text { AND }\end{array}$} & $\begin{array}{r}\text { TESTS } \\
\text { ONCRETE }\end{array}$ \\
\hline & Test & \multicolumn{2}{|c|}{ Same W/C } & \multicolumn{2}{|c|}{$\begin{array}{c}\text { Same } \\
\text { consistance }\end{array}$} \\
\hline & $\begin{array}{l}\text { conc } \\
\text { rete }\end{array}$ & $\begin{array}{l}0.5^{*} \\
\mathrm{Ad} \\
\mathrm{m} .\end{array}$ & $\begin{array}{l}2 \\
A \\
d \\
m\end{array}$ & $\begin{array}{l}0.5 \\
\text { Ad } \\
\mathrm{m} .\end{array}$ & $\begin{array}{c}2 \\
\mathrm{~A} \\
\mathrm{~d} \\
\mathrm{~m} .\end{array}$ \\
\hline $\begin{array}{c}\text { Gravel } \\
\mathrm{s} \\
16 / 31 \\
5 \mathrm{~mm}\end{array}$ & $\begin{array}{l}750 \\
\mathrm{Kg}\end{array}$ & $750 \mathrm{Kg}$ & $\begin{array}{c}750 \mathrm{~K} \\
\mathrm{~g}\end{array}$ & $750 \mathrm{Kg}$ & $750 \mathrm{Kg}$ \\
\hline $\begin{array}{c}\text { Gravel } \\
\mathrm{s} \\
4 / 16 \mathrm{~m} \\
\mathrm{~m}\end{array}$ & $\begin{array}{l}455 \\
\mathrm{Kg}\end{array}$ & $455 \mathrm{Kg}$ & $\begin{array}{l}455 \\
\mathrm{Kg}\end{array}$ & $\begin{array}{l}455 \\
\mathrm{Kg}\end{array}$ & $\begin{array}{l}455 \\
\mathrm{Kg}\end{array}$ \\
\hline $\begin{array}{l}\text { Crushi } \\
\text { ng } \\
\text { sand.0/ } \\
4 \mathrm{~mm}\end{array}$ & $\begin{array}{l}390 \\
\mathrm{Kg}\end{array}$ & $390 \mathrm{Kg}$ & $\begin{array}{l}390 \\
\mathrm{Kg}\end{array}$ & $\begin{array}{l}390 \\
\mathrm{Kg}\end{array}$ & $\begin{array}{l}390 \\
\mathrm{Kg}\end{array}$ \\
\hline $\begin{array}{l}\text { Dredgi } \\
\text { ng } \\
\text { Sand }\end{array}$ & $\begin{array}{c}390 \\
\mathrm{Kg}\end{array}$ & $390 \mathrm{Kg}$ & $\begin{array}{l}390 \\
\mathrm{Kg}\end{array}$ & $\begin{array}{l}390 \\
\mathrm{Kg}\end{array}$ & $\begin{array}{l}390 \\
\mathrm{Kg}\end{array}$ \\
\hline
\end{tabular}




\begin{tabular}{|c|c|c|c|c|c|}
\hline $\begin{array}{c}0 / 0.63 \\
\mathrm{~mm}\end{array}$ & & & & & \\
\hline Cemen & 300 & $300 \mathrm{Kg}$ & 300 & 300 & 300 \\
\hline $\begin{array}{c}\mathrm{t} \\
\text { CPJ55 } \\
\text { PM }\end{array}$ & $\mathrm{Kg}$ & & $\mathrm{Kg}$ & $\mathrm{Kg}$ & $\mathrm{Kg}$ \\
\hline $\begin{array}{l}\text { Total } \\
\text { water }\end{array}$ & $\begin{array}{c}163 \\
\mathrm{~L}\end{array}$ & $161 \mathrm{~L}$ & $\begin{array}{c}157 \\
\mathrm{~L}\end{array}$ & $149 \mathrm{~L}$ & $114 \mathrm{~L}$ \\
\hline $\begin{array}{l}\text { Viscoc } \\
\text { rète } \\
\text { Tempo } \\
1000 \mathrm{M}\end{array}$ & -- & $1.65 \mathrm{Kg}$ & $\begin{array}{l}6.6 \\
\mathrm{Kg}\end{array}$ & $\begin{array}{l}1.65 \\
\mathrm{Kg}\end{array}$ & $6.6 \mathrm{Kg}$ \\
\hline $\mathrm{W} / \mathrm{C}$ & 0.50 & 0.50 & 0.50 & 0.47 & 0.35 \\
\hline $\begin{array}{c}\text { Slump } \\
\text { à T0 }\end{array}$ & $\begin{array}{c}30 \mathrm{~m} \\
\mathrm{~m}\end{array}$ & $90 \mathrm{~mm}$ & *NM & $55 \mathrm{~mm}$ & $65 \mathrm{~mm}$ \\
\hline $\begin{array}{c}\text { Densit } \\
\text { y of } \\
\text { fresh } \\
\text { concret } \\
\mathrm{e} \\
(\mathrm{T} / \mathrm{m} 3)\end{array}$ & 2.40 & 2.41 & 2.40 & 2.42 & $\begin{array}{l}2 . \\
45\end{array}$ \\
\hline $\begin{array}{c}\text { Air } \\
\text { content } \\
(\%)\end{array}$ & 3.1 & 2.6 & 2.3 & 2.4 & $\begin{array}{l}1 \\
9\end{array}$ \\
\hline $\begin{array}{c}\mathrm{T}^{\circ} \\
\text { Concre } \\
\text { te }\end{array}$ & 23.2 & 23.6 & 24.1 & 23.7 & $\begin{array}{l}23 \\
.0\end{array}$ \\
\hline $\begin{array}{l}\mathrm{T}^{\circ} \text { of } \\
\text { constit } \\
\text { uents } \\
\left({ }^{\circ} \mathrm{C}\right)\end{array}$ & \multicolumn{5}{|c|}{$\begin{array}{c}\text { water }=20.8 ; \mathrm{SC} 0 / 4=22.1 ; \mathrm{SD}=23.2 ; \\
\text { Gravels }=22.0 ; \\
\mathrm{T}^{\circ} \mathrm{Admixture}=21.7 ; \mathrm{T}^{\circ} \mathrm{Cement}=23.9\end{array}$} \\
\hline $\begin{array}{c}\mathrm{RC} a ̀ \\
3 \mathrm{j} \\
(\mathrm{MPa})\end{array}$ & 13.7 & 15.3 & 18.4 & 19.6 & $\begin{array}{l}32 \\
.8\end{array}$ \\
\hline $\begin{array}{c}\mathrm{RC} a ̀ \\
28 \mathrm{j} \\
(\mathrm{MPa})\end{array}$ & 32.8 & 34.1 & 36.7 & 38.2 & $\begin{array}{l}54 \\
.4\end{array}$ \\
\hline
\end{tabular}

\subsection{Nominal composition of the studied concrete formulations of tetrapods based on dredged sand from the port of Agadir}

The composition of the studied concrete formulation was carried out by the method of Dreux Goriss, thus three formulations were carried out to find the composition of each constituent of the concrete of the tetrapods based on dredged sand and using a 3rd generation admixture to significantly improve the results found.
Table 8: Nominal COMPOSITION OF THE STUDied CONCRETE FORMULATIONS OF TETRAPODS BASED ON DREDGED SAND

\begin{tabular}{|c|c|c|c|}
\hline \multirow{2}{*}{ Designation } & \multicolumn{3}{|c|}{ Concrete $\left(\mathrm{d}=2.40 \mathrm{~T} / \mathrm{m}^{3}\right)$} \\
\hline & $\begin{array}{c}\text { Formu } \\
\text { la1 }\end{array}$ & $\begin{array}{c}\text { Formu } \\
\text { la2 }\end{array}$ & $\begin{array}{c}\text { Formu } \\
\text { la3 }\end{array}$ \\
\hline $\begin{array}{c}\text { Gravels } \\
16 / 31.5 \mathrm{~mm}\end{array}$ & $750 \mathrm{Kg}$ & $750 \mathrm{Kg}$ & $750 \mathrm{Kg}$ \\
\hline $\begin{array}{l}\text { Gravels } \\
4 / 16 \mathrm{~mm}\end{array}$ & $455 \mathrm{Kg}$ & $455 \mathrm{Kg}$ & $455 \mathrm{Kg}$ \\
\hline S.C. $0 / 4 \mathrm{~mm}$ & $390 \mathrm{Kg}$ & $390 \mathrm{Kg}$ & $390 \mathrm{Kg}$ \\
\hline $\begin{array}{l}\text { Dredging } \\
\text { Sand }\end{array}$ & $390 \mathrm{Kg}$ & $390 \mathrm{Kg}$ & $390 \mathrm{Kg}$ \\
\hline $\begin{array}{c}\text { CementCPJ5 } \\
\text { 5PM }\end{array}$ & $300 \mathrm{Kg}$ & $300 \mathrm{Kg}$ & $300 \mathrm{Kg}$ \\
\hline Tatal water & $140 \mathrm{~L}$ & $140 \mathrm{~L}$ & $140 \mathrm{~L}$ \\
\hline $\begin{array}{l}\text { Viscocrète } \\
\text { Tempo } \\
1000 \mathrm{M}\end{array}$ & $3.3 \mathrm{Kg}$ & $3.3 \mathrm{Kg}$ & $3.3 \mathrm{Kg}$ \\
\hline $\begin{array}{l}\text { E.eff. / } \\
\text { ciment }\end{array}$ & 0.43 & 0.43 & 0.43 \\
\hline Slump-test T0 & $180 \mathrm{~mm}$ & $170 \mathrm{~mm}$ & $180 \mathrm{~mm}$ \\
\hline $\begin{array}{l}\text { Slump-test } \\
\text { T30 }\end{array}$ & $140 \mathrm{~mm}$ & $130 \mathrm{~mm}$ & $150 \mathrm{~mm}$ \\
\hline $\begin{array}{l}\text { Slump-test } \\
\text { T60 }\end{array}$ & $60 \mathrm{~mm}$ & $60 \mathrm{~mm}$ & $70 \mathrm{~mm}$ \\
\hline $\begin{array}{c}\text { Air occluded } \\
(\%)\end{array}$ & 2.1 & 2.3 & 2.3 \\
\hline $\begin{array}{c}\text { Density of } \\
\text { fresh concrete } \\
(\mathrm{T} / \mathrm{m} 3)\end{array}$ & 2.44 & 2.42 & 2.43 \\
\hline
\end{tabular}

\subsection{Results found for the concretes of the studied formulations}

The results of the hardened concretes of the studied formulations are summarized in the following table:

Table 9: RESUlts OF THE HARDENED CONCRETE OF THE FORMULATIONS STUDIED BASED ON DREDGING SAND

\begin{tabular}{|c|c|c|c|}
\hline \multirow{2}{*}{$\begin{array}{c}\text { Designati } \\
\text { on }\end{array}$} & \multicolumn{3}{|c|}{ Concrete $\left(\mathrm{d}=2.40 \mathrm{~T} / \mathrm{m}^{3}\right)$} \\
\cline { 2 - 4 } & $\begin{array}{c}\text { Formul } \\
\mathrm{a} 1\end{array}$ & $\begin{array}{c}\text { Formul } \\
\mathrm{a} 2\end{array}$ & $\begin{array}{c}\text { Formul } \\
\mathrm{a} 3\end{array}$ \\
\hline $\begin{array}{c}\mathrm{RC} \text { à } 7 \mathrm{j} \\
(\mathrm{MPa})\end{array}$ & 34.1 & 32.7 & 31.9 \\
\hline
\end{tabular}




\begin{tabular}{|c|c|c|c|}
\hline $\begin{array}{c}\text { RC à } 28 \mathrm{j} \\
(\mathrm{MPa})\end{array}$ & 43.9 & 43.2 & 42.6 \\
\hline
\end{tabular}

The arithmetic mean fcE of the 9 measurements (3 measurements per formula) of the compressive strength measured at 28 days shall satisfy the following 2 conditions:

$-\mathrm{fcE}>\mathrm{fc} 28+\left(\mathrm{Ce} \_\mathrm{Cmin}\right)$

$-\mathrm{fcE}>\mathrm{fc} 28+3$

Table 10: Resistance OF THE STUDIED CONCRETE

\begin{tabular}{|c|c|c|c|}
\hline Concrete & fc28 & fcE & Ce \\
\hline $\begin{array}{c}\text { Concrete B25 } \\
\mathrm{d}=2.4 \mathrm{~T} / \mathrm{m}^{3}\end{array}$ & 25 & 43.2 & 51.7 \\
\hline
\end{tabular}

\begin{tabular}{|c|c|c|c|}
\hline Concrete & Cmin & fc28 $+(\mathrm{Ce}-$ Cmin $)$ & fc28+3 \\
\hline $\begin{array}{c}\text { Concrete B25 } \\
\mathrm{d}=2.4 \mathrm{~T} / \mathrm{m}^{3}\end{array}$ & 42.5 & 34.2 & 33 \\
\hline
\end{tabular}

For Concrete B25, we therefore have :

- 43.2 >34.2 MPa conform;

- 43.2 >33 MPa conform.

\section{Conclusion}

The studied formulation based on dredged sand from the port of Agadir in percentage with crushed sand and materials from the region gave good results and characteristics in terms of mechanical resistance, the formulation has shown that it can be used in the fabrication of tetrapods and that they can be used as protection against swells in port protection works.

The use of dredging sand in the production of tetrapods will allow to make a gain in terms of the deposit of construction materials and to develop the resource of dredging sand of the port of Agadir generally considered as waste.

\section{Acknowledgements}

We would like to thank all those who have contributed in one way or another to the realization of this research work.

\section{References}

Moore, R., Lopes, J., 1999. Paper templates. In TEMPLATE'06, 1st International Conference on Template Production. SCITEPRESS.

Smith, J., 1998. The book, The publishing company. London, $2^{\text {nd }}$ edition.

J. Xiao, C. Qiang, A. Nanni, and K. Zhang, "Use of sea-sand and seawater in concrete construction: Current status and future opportunities," Constr. Build. Mater., vol. 155, pp. 1101-1111, 2017. https://doi.org/10.1016/j.conbuildmat.2017.08.130

M. HASSOUNE, G. CHRAIBI, H. FATMAOUI, J.CHAOUFI, 2019. Requalification of dredging sediments through their use in concrete formulations based on a 3rd generation admixture. Materials Today: Proceedings. https://doi.org/10.1016/j.matpr.2019.08.065

Plan qualité de dragage d'entretien du port d'Agadirdrapor / Data from the National Ports Agency at the port of Agadir

P. Ozer-Erdogan, H. M. Basar, I. Erden, and L. Tolun, "Beneficial use of marine dredged materials as a fine aggregate in ready-mixed concrete: Turkey example," Constr. Build. Mater., vol. 124, pp. 690 704 ,

2016. https://doi.org/10.1016/j.conbuildmat.2016.07.144

Girish.C.G, "dredged offshore s and as a replacement for fine," vol. 8, no. 3, pp. 88-95, 2015.

R. Mahendran, K. Godwin, T. G. Selvan, and M. Murugan, "experimental study on concrete using sea sand," vol. 7, no. 5, pp. 49-52, 2016.

G. Dreux, J. Festa, , "Nouveau guide du béton et de ses constituants."

Bathymetric surveys at the port of Agadir, Agence Nationale des Ports.

C. Julien, "Gestion d' un sédiment de dragage marin contaminé : Caractérisation de la réactivité biogéochimique, valorisation en mortier et évaluation environnementale Julien Couvidat To cite this version : HAL Id : tel-01368438," 2016.

C. Sheehan and J. Harrington, "Management of dredge material in the Republic of Ireland - A review," Waste Manag., vol. 32, no. 5, pp. 1031-1044, 2012. https://doi.org/10.1016/j.wasman.2011.11.014

J. Limeira, L. Agullo, and M. Etxeberria, "Dredged marine sand in concrete: An experimental section of a harbor pavement," Constr. Build. Mater., vol. 24, no. $6, \quad$ pp. $863-870, \quad 2010$ https://doi.org/10.1016/j.conbuildmat.2009.12.011

E. C. De Lille and E. C. De Lille, "Inertage et valorisation des sédiments de dragage marins," 2006.

M. G. S. Dias, D. a R. Dolage, and C. T. Ariyawansa, "Offshore Sand as a Fine Aggregate for Concrete Production," vol. 3, no. 4, pp. 813-825, 2013. https://doi.org/10.9734/bjast/2014/3290

J. Mindeguia, P. Pimienta, and C. La Borderie, "European Journal of Environmental and Civil 
Engineering," no. May, pp. 37-41, 2012. https://doi.org/10.1080/19648189.2012.667682

EN 206-1, Norme européenne:béton-partie 1: spécifications, performances, production et conformité, AFNOR, jan.2000.

P 18-542, Fascicule de documentation: Granulats courants pour béton hydraulique-critères de qualification des granulats vis-à-vis de l'alacaliréactions, AFNOR, mai 1994.

Dragage en milieu marin, immersion et code de l'environnement: le guide des procédures préalables.

R. Polder, "Durability of marine concrete structures_field investigations and modellling,".

Northern Ireland Environment Agency and Scottish Environment Protection Agency, "Interpretation of the definition and classification of hazardous waste," 2005.

A.R. Roeder, Some technical data on sea-dredged aggregates, in: Proceedings of the Symposium: Seadredged Aggregates for Concrete. Sand and GravelAssociation Great Britain, Buckinghamshire, UK, 1968.

S. Marmin, J. Dauvin, P. Lesueur, Ocean\&coastal management collaborative approach for the management of harbour-dredged sediment in the Bay of Seine (France), Ocean Coast. Manage. 102 (2014) $328-339$ http://dx.doi.org/10.1016/j.ocecoaman.2014.10.012. For papers published in translation journals, please give the English citation first, followed by the original foreign-language citation . 